\title{
Are There Infinitely Many twin Primes?
}

\section{Edigles Guedes ${ }^{1}$, Prof. Dr. Raja Rama Gandhi ${ }^{2}$ and Srinivas Kishan Anapu ${ }^{3}$ \\ ${ }^{1}$ Number Theorist, Pernambuco, Brazil. \\ ${ }^{2}$ Resource person in Math for Oxford University Press, Professor in Math, BITS-Vizag. \\ ${ }^{3}$ CEO, Voice of Bigdata, Austin, TX.ABSTRACT.}

Keywords: Rosser's theorem, Dusart's inequality

ABSTRACT. We prove that are there infinitely many twin primes.

\section{INTRODUCTION}

One of the most famous and beautiful open problem in number theory is the twin prime conjecture: are there infinitely many twin primes?

In this paper, we demonstrate that yes, using an analytic proof by contradiction, Rosser's theorem and Dusart's inequality.

\section{PRELIMINARES}

The Rosser's theorem [1] states that $p_{n}$ is $\operatorname{larger}$ than $n \log n$. This can be improved by the following pair of bounds:

$$
\log n+\log \log n-1<\frac{p_{n}}{n}<\log n+\log \log n,
$$

for $n \geq 6$.

\section{THEOREM}

THEOREM 1. Are there infinitely many twin primes.

Proof. We will use the reductio ad impossibilem. Hence, we assume, by hypothesis, which are there finitely many twin prime numbers.

Step 1. We let, by Rosser's theorem,

$$
\log (n+1)+\log \log (n+1)-1<\frac{p_{n+1}}{n+1}<\log (n+1)+\log \log (n+1)
$$

and

$$
\log n+\log \log n-1+\frac{2}{n}<\frac{p_{n}+2}{n}<\log n+\log \log n+\frac{2}{n}
$$

for $n \geq 6$.

Dividing (3.1) by (3.2), member by member, we have

$$
\frac{\log (n+1)+\log \log (n+1)-1}{\log n+\log \log n-1+\frac{2}{n}}<\frac{p_{n+1}}{p_{n}+2} \cdot \frac{n}{n+1}<\frac{\log (n+1)+\log \log (n+1)}{\log n+\log \log n+\frac{2}{n}},
$$

Multiplying (3.3) by $\frac{n+1}{n}$, we encounter

$$
\frac{n+1}{n} \cdot \frac{\log (n+1)+\log \log (n+1)-1}{\log n+\log \log n-1+\frac{2}{n}}<\frac{p_{n+1}}{p_{n}+2}<\frac{n+1}{n} \cdot \frac{\log (n+1)+\log \log (n+1)}{\log n+\log \log n+\frac{2}{n}}
$$




$$
(n+1) \cdot \frac{\log (n+1)+\log \log (n+1)-1}{n \log n+n \log \log n-n+2}<\frac{p_{n+1}}{p_{n}+2}<(n+1) \cdot \frac{\log (n+1)+\log \log (n+1)}{n \log n+n \log \log n+2}
$$

From (3.4), we have

$$
n+1<\frac{p_{n+1}}{p_{n}+2} \cdot \frac{n \log n+n \log \log n-n+2}{\log (n+1)+\log \log (n+1)-1}
$$

and

$$
\frac{p_{n+1}}{p_{n}+2} \cdot \frac{n \log n+n \log \log n+2}{\log (n+1)+\log \log (n+1)}<n+1 .
$$

Inverting (3.5) and (3.6), we obtain

$$
\frac{p_{n}+2}{p_{n+1}} \cdot \frac{\log (n+1)+\log \log (n+1)-1}{n \log n+n \log \log n-n+2}<\frac{1}{n+1}
$$

and

$$
\frac{1}{n+1}<\frac{p_{n}+2}{p_{n+1}} \cdot \frac{\log (n+1)+\log \log (n+1)}{n \log n+n \log \log n+2} .
$$

We set $n+1 \rightarrow n$ in (3.7) and (3.8)

$$
\frac{p_{n-1}+2}{p_{n}} \cdot \frac{\log n+\log \log n-1}{(n-1) \log (n-1)+(n-1) \log \log (n-1)-n+3}<\frac{1}{n}<\frac{p_{n-1}+2}{p_{n}} \cdot \frac{\log n+\log \log n}{(n-1) \log (n-1)+(n-1) \log \log (n-1)+2}
$$

The summation in $n$ from 6 to infinity, give us

$\sum_{n=6}^{\infty} \frac{p_{n-1}+2}{p_{n}} \cdot \frac{\log n+\log \log n-1}{(n-1) \log (n-1)+(n-1) \log \log (n-1)-n+3}<\sum_{n=6}^{\infty} \frac{1}{n}<\sum_{n=6}^{\infty} \frac{p_{n-1}+2}{p_{n}}$. $\log n+\log \log n$

$(n-1) \log (n-1)+(n-1) \log \log (n-1)+2$.

Summing $\sum_{n=1}^{5} \frac{1}{n}$ in each member of (3.10), we find

$$
\begin{aligned}
& \sum_{n=1}^{5} \frac{1}{n}+\sum_{n=6}^{\infty} \frac{p_{n-1}+2}{p_{n}} \cdot \frac{\log n+\log \log n-1}{(n-1) \log (n-1)+(n-1) \log \log (n-1)-n+3} \\
< & \sum_{n=1}^{\infty} \frac{1}{n}<\sum_{n=1}^{5} \frac{1}{n}+\sum_{n=6}^{\infty} \frac{p_{n-1}+2}{p_{n}} \cdot \frac{\log n+\log \log n}{(n-1) \log (n-1)+(n-1) \log \log (n-1)+2},
\end{aligned}
$$

so

$$
\begin{gathered}
H_{5}+\sum_{n=6}^{\infty} \frac{p_{n-1}+2}{p_{n}(n-1)} \cdot \frac{\log n+\log \log n-1}{\log (n-1)+\log \log (n-1)-\left(\frac{n-3}{n-1}\right)} \\
<\sum_{n=1}^{\infty} \frac{1}{n}<H_{5}+\sum_{n=6}^{\infty} \frac{p_{n-1}+2}{p_{n}(n-1)} \cdot \frac{\log n+\log \log n}{\log (n-1)+\log \log (n-1)+\frac{2}{n-1}},
\end{gathered}
$$

where $H_{n}$ denote the $n$-th harmonic number.

The maximum of $\frac{\log n+\log \log n}{\log (n-1)+\log \log (n-1)+\frac{2}{n-1}}$, for $n \in \mathbb{N}_{\geq 6}$, is $\lim _{n \rightarrow \infty} \frac{\log n+\log \log n}{\log (n-1)+\log \log (n-1)+\frac{2}{n-1}}=$ 1. In other words, 


$$
\begin{array}{r}
\max _{n \in \mathbb{N}_{\geq 6}} \frac{\log n+\log \log n}{\log (n-1)+\log \log (n-1)+\frac{2}{n-1}} \\
=\lim _{n \rightarrow \infty} \frac{\log n+\log \log n}{\log (n-1)+\log \log (n-1)+\frac{2}{n-1}}=1 .
\end{array}
$$

The minimum of $\frac{\log n+\log \log n-1}{\log (n-1)+\log \log (n-1)-\left(\frac{n-3}{n-1}\right)}, \quad$ for $\quad n \in \mathbb{N}_{\geq 6}$, is $\frac{\log 6+\log \log 6-1}{\log 5+\log \log 5-\frac{3}{5}}=0.925696050889805 \ldots$ In other words,

$$
\begin{gathered}
\min _{n \in \mathbb{N}_{\geq 6}} \frac{\log n+\log \log n-1}{\log (n-1)+\log \log (n-1)-\left(\frac{n-3}{n-1}\right)} \\
=\frac{\log 6+\log \log 6-1}{\log 5+\log \log 5-\frac{3}{5}}=0.925696050889805 \ldots=: C_{1}
\end{gathered}
$$

From (3.11), (3.12) and (3.13), we encounter

$$
\begin{gathered}
H_{5}+C_{1} \sum_{n=6}^{\infty} \frac{p_{n-1}+2}{p_{n}(n-1)}=H_{5}+C_{1}\left[\sum_{n=6}^{\infty} \frac{p_{n-1}}{p_{n}(n-1)}+\sum_{n=6}^{\infty} \frac{2}{p_{n}(n-1)}\right]<\sum_{n=1}^{\infty} \frac{1}{n}< \\
H_{5}+\sum_{n=6}^{\infty} \frac{p_{n-1}+2}{p_{n}(n-1)}=H_{5}+\left[\sum_{n=6}^{\infty} \frac{p_{n-1}}{p_{n}(n-1)}+\sum_{n=6}^{\infty} \frac{2}{p_{n}(n-1)}\right] .
\end{gathered}
$$

In [2], we have

$$
\sum_{n=1}^{\infty} \frac{1}{n}=\prod_{i=1}^{\infty} \frac{1}{1-p_{i}^{-1}}=\frac{1}{1-p_{1}^{-1}} \cdot \frac{1}{1-p_{2}^{-1}} \cdot \frac{1}{1-p_{3}^{-1}} \cdot \frac{1}{1-p_{4}^{-1}} \cdot \frac{1}{1-p_{5}^{-1}} \cdot \ldots
$$

We can factor (3.15) in prime numbers that are not twin primes and twin primes without repetition in each member, that is, not a pair of twin primes, just every twin prime number. Explicitly, $\quad \widetilde{\mathbb{P}}_{\text {twin }}=\{2,23,31,37,47,53,67,79, \ldots\} \quad$ and $\quad \mathbb{P}_{\text {twin }}=\{3,5,7,11,13,17,19,29,31, \ldots\}$. After, it follows that

$$
\sum_{n=1}^{\infty} \frac{1}{n}=\prod_{i=1}^{\infty} \frac{1}{1-p_{i}^{-1}}=\prod_{p \in \widetilde{\mathbb{P}}_{\mathrm{twin}}} \frac{1}{1-p^{-1}} \cdot \prod_{p \in \mathbb{P}_{\mathrm{twin}}} \frac{1}{1-p^{-1}}
$$

Substituting (3.16) in (3.14), we obtain

$H_{5}+C_{1}\left[\sum_{n=6}^{\infty} \frac{p_{n-1}}{p_{n}(n-1)}+\sum_{n=6}^{\infty} \frac{2}{p_{n}(n-1)}\right]<\sum_{n=1}^{\infty} \frac{1}{n}=\prod_{i=1}^{\infty} \frac{1}{1-p_{i}^{-1}}=\prod_{p \in \widetilde{\mathbb{P}}_{\text {twin }}} \frac{1}{1-p^{-1}}$.

$\prod_{p \in \mathbb{P}_{\text {twin }}} \frac{1}{1-p^{-1}}<H_{5}+\left[\sum_{n=6}^{\infty} \frac{p_{n-1}}{p_{n}(n-1)}+\sum_{n=6}^{\infty} \frac{2}{p_{n}(n-1)}\right]$.

Multiplying both members of (3.17) by $\prod_{p \in \widetilde{\mathbb{P}}_{\text {twin }}}\left(1-p^{-1}\right)$, then

$$
\begin{aligned}
& \prod_{p \in \widetilde{\mathbb{P}}_{\text {twin }}}\left(1-p^{-1}\right) \cdot\left\{H_{5}+C_{1}\left[\sum_{n=6}^{\infty} \frac{p_{n-1}}{p_{n}(n-1)}+\sum_{n=6}^{\infty} \frac{2}{p_{n}(n-1)}\right]\right\}<\prod_{p \in \widetilde{\mathbb{P}}_{\text {twin }}}\left(1-p^{-1}\right) \cdot \sum_{n=1}^{\infty} \frac{1}{n} \\
= & \prod_{p \in \mathbb{P}_{\text {twin }}} \frac{1}{1-p^{-1}}<\prod_{p \in \widetilde{\mathbb{P}}_{\text {twin }}}\left(1-p^{-1}\right) \cdot\left\{H_{5}+\left[\sum_{n=6}^{\infty} \frac{p_{n-1}}{p_{n}(n-1)}+\sum_{n=6}^{\infty} \frac{2}{p_{n}(n-1)}\right]\right\} .(3.18
\end{aligned}
$$

Step 2. We prove that the series

$$
\sum_{n=6}^{\infty} \frac{2}{p_{n}(n-1)}
$$


is divergent. By Dusart's inequality [2], we set

$$
p_{n}<n \log n+n \log \log n,
$$

for $n \geq 6$. Thus,

$$
\begin{aligned}
\sum_{n=6}^{\infty} \frac{2}{p_{n}(n-1)}=2 \sum_{n=6}^{\infty} & \frac{1}{p_{n}(n-1)} \geq 2 \sum_{n=6}^{\infty} \frac{1}{(n \log n+n \log \log n)(n-1)} \\
& \geq 2 \sum_{n=6}^{\infty} \frac{1}{2 n \log n(n-1)}=\sum_{n=6}^{\infty} \frac{1}{n \log n(n-1)} \\
= & \sum_{n=6}^{\infty}\left\{\frac{1}{n \log n}-\left[\frac{n}{n(n-1) \log n}-\frac{2}{n(n-1) \log n}\right]\right\} \\
& =\sum_{n=6}^{\infty}\left[\frac{1}{n \log n}-\frac{1}{(n-1) \log n}+\frac{2}{n(n-1) \log n}\right] \\
= & \sum_{n=6}^{\infty} \frac{1}{n \log n}-\sum_{n=6}^{\infty} \frac{1}{(n-1) \log n}+\sum_{n=6}^{\infty} \frac{2}{n(n-1) \log n} .
\end{aligned}
$$

Note 1: is easy to check that $\sum_{n=6}^{\infty} \frac{1}{n \log n}$ is divergent by the integral test of convergence, $\sum_{n=6}^{\infty} \frac{1}{(n-1) \log n}$ is divergent by the integral test of convergence, and $\sum_{n=6}^{\infty} \frac{2}{n(n-1) \log n}$ is divergent by the root test of convergence. This demonstrate that the series on the left diverges.

Step 3. We prove that the series

$$
\sum_{n=6}^{\infty} \frac{p_{n-1}}{p_{n}(n-1)}
$$

is divergent. By Rosser's inequality [1], we get

$$
n \log n+n \log \log n-n<p_{n}<n \log n+n \log \log n,
$$

for $n \geq 6$. Thus,

$$
\begin{gathered}
\sum_{n=6}^{\infty} \frac{p_{n-1}}{p_{n}(n-1)} \geq \sum_{n=6}^{\infty} \frac{(n-1) \log (n-1)+(n-1) \log \log (n-1)-n+1}{(n \log n+n \log \log n)(n-1)} \\
=\sum_{n=6}^{\infty} \frac{\log (n-1)+\log \log (n-1)-1}{n \log n+n \log \log n} \geq \sum_{n=6}^{\infty} \frac{\log (n-1)+\log \log (n-1)-1}{2 n \log n} \\
=\sum_{n=6}^{\infty}\left[\frac{\log (n-1)}{2 n \log n}+\frac{\log \log (n-1)}{2 n \log n}-\frac{1}{2 n \log n}\right] \\
=\frac{1}{2}\left[\sum_{n=6}^{\infty} \frac{\log (n-1)}{n \log n}+\sum_{n=6}^{\infty} \frac{\log \log (n-1)}{n \log n}-\sum_{n=6}^{\infty} \frac{1}{n \log n}\right] .
\end{gathered}
$$

Note 2: is easy to verify that $\sum_{n=6}^{\infty} \frac{\log (n-1)}{n \log n}$ is divergent by the root test of convergence, $\sum_{n=6}^{\infty} \frac{\log \log (n-1)}{n \log n}$ is divergent by the root test of convergence, and that $\sum_{n=6}^{\infty} \frac{1}{n \log n}$ is divergent by the integral test of convergence. This demonstrate that the series on the left diverges. 
Step 4. Note 3 : is easy to verify that $\sum_{n=1}^{\infty} \frac{1}{n}$ is divergent by the integral test of convergence.

Step 5. By hypothesis, we note that $\prod_{p \in \mathbb{P}_{\text {twin }}} \frac{1}{1-p^{-1}}$ is convergent, since $\mathbb{P}_{\text {twin }}$ is finite. But, from (3.18), Conclusion 1, Conclusion 2 and Conclusion 3, we note that the left hand side and the right hand side of inequality (3.18) is divergent, independently if $\prod_{p \in \widetilde{\mathbb{P}}_{\text {twin }}}\left(1-p^{-1}\right)$ is convergent or divergent; and the left hand side of equality also is divergent, independently if $\prod_{p \in \widetilde{\mathbb{P}}_{\text {twin }}}\left(1-p^{-1}\right)$ is convergent or divergent. This is an absurd! The contradiction that we wanted is guaranteed. Thus, are there infinitely many twin prime numbers.

\section{OPEN QUESTION}

The first author, Edigles Guedes, leave to Prof. Dr. Raja Rama Gandhi the following problem:

$$
\prod_{p \in \widetilde{\mathbb{P}}_{\text {twin }}}\left(1-p^{-1}\right)
$$

is convergent or divergent? what its value asymptotic?

\section{ACKNOWLEDGMENTS}

The first author, Edigles Guedes, thank Prof. Dr. K. Raja Rama Gandhi by her encouragement and support during the development of this paper.

\section{REFERENCES}

[1] http://en.wikipedia.org/wiki/Prime_number_theorem, available in May 11, 2013.

[2] http://en.wikipedia.org/wiki/Proof_that_the_sum_of_the_reciprocals_of_the_primes_diverges, available in June 5, 2013. 